\title{
The Research of Car-Following Model Based on Real-Time Maximum Deceleration
}

\author{
Longhai Yang, Xiqiao Zhang, Jiekun Gong, and Juntao Liu \\ School of Transportation Science and Engineering, Harbin Institute of Technology, Harbin 150090, China \\ Correspondence should be addressed to Jiekun Gong; gongjiekun@126.com
}

Received 3 April 2015; Accepted 18 June 2015

Academic Editor: Yuanchang Xie

Copyright (C) 2015 Longhai Yang et al. This is an open access article distributed under the Creative Commons Attribution License, which permits unrestricted use, distribution, and reproduction in any medium, provided the original work is properly cited.

This paper is concerned with the effect of real-time maximum deceleration in car-following. The real-time maximum acceleration is estimated with vehicle dynamics. It is known that an intelligent driver model (IDM) can control adaptive cruise control (ACC) well. The disadvantages of IDM at high and constant speed are analyzed. A new car-following model which is applied to ACC is established accordingly to modify the desired minimum gap and structure of the IDM. We simulated the new car-following model and IDM under two different kinds of road conditions. In the first, the vehicles drive on a single road, taking dry asphalt road as the example in this paper. In the second, vehicles drive onto a different road, and this paper analyzed the situation in which vehicles drive from a dry asphalt road onto an icy road. From the simulation, we found that the new car-following model can not only ensure driving security and comfort but also control the steady driving of the vehicle with a smaller time headway than IDM.

\section{Introduction}

Driving assistance systems and vehicle-sensor designs are becoming mature along with the development of intelligent transportation. Adaptive cruise control (ACC), which is a component of driving assistance systems, is gradually replacing part of the driving control of the human driver. ACC not only alleviates driving fatigue but also improves driving safety. The operation of ACC depends on the information on the vehicle motion and the external environment, which is detected by vehicle sensors. Therefore, reasonable and effective use of sensor data can improve the car-following safety and stability of ACC and can improve the road capacity effectively.

The tire-road friction coefficient, which determines the real-time maximum deceleration, is a significant parameter for ensuring the vehicle driving safely and avoiding rearend collisions. The estimation methods can be divided into two types: direct detection with sensors and vehicle dynamic model estimation. Direct detection methods, such as detecting the coefficient with optical or acoustic sensors, need expensive sensors which limit the use of the method [1]. Its reliability and robustness is low, because the detection precision varies with the environment. The estimation of the coefficient with the vehicle dynamic model has higher robustness, but the accuracy still needs to be improved [2]. In order to improve the estimation accuracy of the latter method, the combination of a vehicle dynamic model with data fusion of multiple sensors has become a hot topic of research [3]. Recently, estimation methods with vehicle dynamic models, which include slip-slope methods, Kalman filter-based friction coefficient estimation, and methods based on lateral dynamics, have concentrated on estimating the mean coefficient value of four wheels [4]. However, the latest researches indicate that friction coefficient estimation of individual wheels has greater value [5].

The friction coefficient is used in vehicle collisionavoidance systems, antilock braking systems (ABS), and electronic stability systems (ESC). The real-time friction coefficient has important significance for influencing the carfollowing behavior and guaranteeing traffic safety. But there are few researches which have included the real-time friction coefficient in car-following models until now. Most of the traditional car-following models regard the maximum deceleration as a constant value and lack consideration of the realtime road conditions [6]. In the following controls of ACC and CACC, the driver can choose different modes according to the real-time driving environment that the driver can 


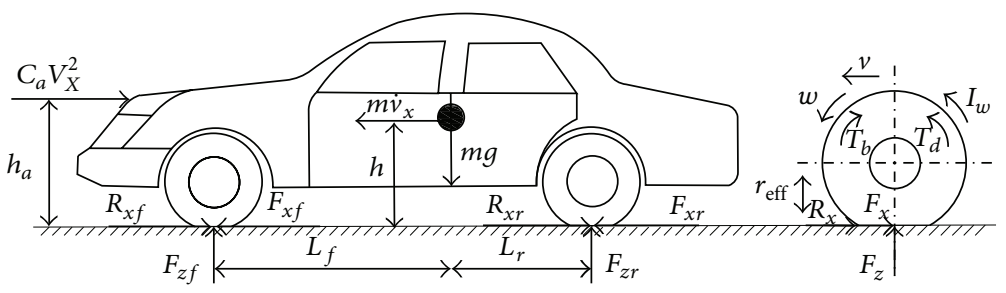

FIGURE 1: Longitudinal vehicle dynamics schematic.

perceive. Different modes have corresponding gaps. For the ACC system, the available gap settings are 1.1, 1.6, and $2.2 \mathrm{~s}$. For the CACC system, the available gap settings are 0.6, 0.9, and 1.1s [7]. The gap controls of ACC and CACC ignore differentiation between different vehicles on the same road. Therefore, the gap should be optimized based on the detected road condition.

In this paper, we propose a new car-following model based on the maximum deceleration, which is estimated with the vehicle dynamic model. This paper is organized as follows. The study of the car-following model in real-time conditions is presented in Section 2. In Section 3, the approach of real-time friction coefficient estimation is introduced. In Section 4, the maximum deceleration is calculated according to the longitudinal vehicle model. The new car-following model is established in Section 5 and simulated in Section 6.

\section{Real-Time Conditions in Car-Following Model}

Generally, the road condition is defined as a congested road or an uncongested road. The car-following models have different parameter values, which are calibrated by experimental data, in the different road conditions [8]. Kesting et al. summarized traffic situations as free traffic, upstream front, congested traffic, bottleneck, and downstream front [9]. The car-following model detected the real-time traffic situation and used corresponding parameters, which differed under different situations, to avoid congestion actively.

Soria et al. [10] assessed four car-following models with field data which were collected under different traffic (congested versus uncongested) and weather conditions (rain versus clear sky). The four car-following models were Gipps, Pitt, MITSIM, and modified Pitt. The parameters of the four car-following models were calibrated by field data, and the corresponding parameters were applied to evaluate the simulation precision of four car-following models under different traffic and weather conditions.

Tang et al. [11, 12] developed a car-following model to study the influence of real-time road conditions on driving behavior. The real-time road conditions, which were divided into good, moderate, and bad, were regarded as a separate parameter modifying the full velocity difference (FVD) model. A good road was defined as having no influential factors along the road (e.g., expressway and freeway). A bad road was defined as having many influential factors along the road (e.g., bus station, sidewalk).

The above works analyzed the effect of real-time traffic, weather, and road conditions on car-following by adjusting the parameters of the models. But the real-time road surface conditions were omitted. The real-time road surface condition can be expressed as the tire-road friction coefficient or the theoretical maximum deceleration. In ACC and collisionavoidance systems, the tire-road friction coefficient is used to adjust the braking stance [13]. In the research on carfollowing, only the collision-avoidance model takes into account the real-time road surface condition $[14,15]$. The theoretical maximum deceleration is used to calculate the safe following distance.

\section{Friction Coefficient Estimation}

3.1. Longitudinal Vehicle Model. A longitudinal vehicle model provides theoretical support for individual wheel friction coefficient estimation. In this paper, we assume that the vehicle performs a linear motion on a level road and other degrees of freedom of motion are ignored. The static force models of the vehicle are shown in Figure 1. by

The equations of the longitudinal vehicle model are given

$$
\begin{aligned}
m \dot{v}_{x} & =F_{x}-R_{x}-C_{a} v_{x}^{2}, \\
I_{\omega} \dot{\omega}_{i} & =T_{d i}-T_{b i}-r_{\mathrm{eff}} F_{x i}, \\
F_{z f} & =\frac{m g L_{r}-m \dot{v}_{x} h-C_{a} v_{x}^{2} h_{a}}{L}, \\
F_{z r} & =\frac{m g L_{f}+m \dot{v}_{x} h+C_{a} v_{x}^{2} h_{a}}{L}, \\
s_{i} & =\frac{\left|\omega_{i} r_{\mathrm{eff}}-v_{x}\right|}{\max \left(\omega_{i} r_{\mathrm{eff}}, v_{x}\right)}, \\
\gamma & =\frac{F_{x i}}{F_{z i}},
\end{aligned}
$$

where $m$ is the mass of the vehicle, $i=f l, f r, r l, r r$ are used to separately represent the four wheels of the vehicle, $v_{x}$ is the longitudinal speed, $F_{x}$ is the total longitudinal tire force, which is the summation of the tire forces generated at four tires, $F_{z i}$ is the normal forces of each wheel, $F_{z f l}=F_{z f r}=$ $F_{z f} / 2, F_{z r l}=F_{z r r}=F_{z r} / 2, R_{x}$ is the rolling resistance, $C_{a}$ is an aerodynamic drag parameter, $I_{\omega}$ is the rotational dynamics of each wheel, $\omega$ is the wheel speed, $T_{d}$ and $T_{b}$ represent the drive and brake torques, $r_{\text {eff }}$ represents the effective radius of the tire, $L=L_{f}+L_{r}$ is the wheelbase, $L_{f}$ represents the horizontal distance between the center of gravity and the front wheel, $L_{r}$ represents the horizontal distance between the center of 
gravity and the rear wheel, $h$ and $h_{a}$ are the heights of the center of gravity and wind loading above the ground, and $\gamma$ is the normalized traction force of the tire.

The slip ratio $s_{i}$ is calculated by (5). During braking, $v_{x}$, which is greater than $\omega_{i} r_{\text {eff }}$, is used as the denominator of (5). During acceleration, $\omega_{i} r_{\text {eff }}$, which is greater than $v_{x}$, is used as the denominator of (5) [5].

The longitudinal tire force varies with the slip ratio, friction coefficient, and normal force. In some researches on ABS, in order to improve the efficiency of control and to shorten the braking distance, the slip ratio is adjusted by ABS to maximize the normalized traction force of the tire [16].

3.2. Friction Coefficient Calculation. The approach which is used in this paper to estimate the individual wheel friction coefficient consists of the following three steps:

(1) estimating the longitudinal tire force;

(2) measuring the longitudinal slip ratio at the wheel;

(3) using a recursive least-squares parameter identification algorithm to calculate the tire-road friction coefficient.

The approaches of longitudinal tire force and longitudinal slip ratio estimation mainly perform friction estimation using GPS and torque measurements, torque measurements and an accelerometer, and GPS and an accelerometer. The first approach has greater estimation accuracy than the other two approaches [1]. Therefore, the first approach is utilized to estimate the longitudinal tire force and longitudinal slip ratio in this paper.

In a small slip ratio interval, where the slip ratio is smaller than 0.15 and when the slip ratio is greater than 0.005 , the normalized traction force has a linear relationship with the slip ratio. For any given road, this linear relationship in the range of a small slip ratio is established [1]

$$
\gamma=\frac{F_{x}}{F_{z}}=K s
$$

$K$ presents the slip-slope, whose value changes with the road surface condition and the type of tire. According to previous experimental data, it is found that the tire-road friction coefficient has a linear relationship with the slip-slope [5]. This linear relationship is shown as

$$
u=A K+C
$$

where $A=0.026$ is the proportionality constant and $C=$ 0.047 is a bias constant.

According to (8), we know that the slip-slope is the key to estimate the tire-road friction coefficient and its estimation accuracy determines the estimation accuracy of the tire-road friction coefficient. Consequently, a recursive least-squares parameter identification algorithm is utilized to improve the estimation accuracy.

\section{Maximum Deceleration Estimation}

ESC can make each wheel reach the peak of the normalized traction force by achieving the best slip ratio. The tire-road friction coefficients of the front and rear wheels correspond to $u_{f}$ and $u_{r}$. We assume that the vehicle brakes with the maximum deceleration that the vehicle can attain during emergency braking on a given road. Combining (1), (3), (4), and (6), the following equation can be obtained:

$$
\begin{aligned}
& \frac{u_{r}}{L}\left(m g L_{f}+m a_{\max } h+C_{a} v_{x}^{2} h_{a}\right) \\
& \quad+\frac{u_{f}}{L}\left(m g L_{r}-m a_{\max } h-C_{a} v_{x}^{2} h_{a}\right)-R_{x}-C_{a} v_{x}^{2} \\
& \quad=m a_{\max } .
\end{aligned}
$$

Simplifying (9), the maximum deceleration can be presented as

$$
\begin{aligned}
a_{\max }= & \frac{L_{r} u_{f}+L_{f} u_{r}}{L+h\left(u_{f}-u_{r}\right)} g \\
& +\frac{C_{a} v_{x}^{2} h_{a}\left(u_{r}-u_{f}\right)-R_{x} L-C_{a} v_{x}^{2} L}{m\left[L+h\left(u_{f}-u_{r}\right)\right]} .
\end{aligned}
$$

The air resistance and the rolling resistance account for only a small proportion of the longitudinal force. Ignoring the air resistance and the rolling resistance, the maximum deceleration can be presented as

$$
a_{\max }=\frac{L_{r} u_{f}+L_{f} u_{r}}{L+h\left(u_{f}-u_{r}\right)} g .
$$

\section{Car-Following Model}

The change of real-time maximum deceleration is not significant when a vehicle is steering in a similar section. It is difficult for the driver to judge a subtle change in maximum acceleration through his or her own senses and to adjust the following gap through a subtle change. However, a computer can detect subtle changes of maximum acceleration and then control the throttle valve and brake valve. Consequently, the car-following model considered the real-time maximum deceleration should be applied in ACC or automatic driving. Automatic driving technology is still in its infancy. ACC technology has gradually become mature. In the current study of ACC, the vehicle controls its speed according to the distance and the relative speed between itself and leading vehicle [17]. The ACC system designs different following gaps which the driver can manually select, but its presupposed gap value is constant [7]. The ACC system fails to automatically calculate a more reasonable desired gap according to the realtime road surface conditions.

In order to guarantee computational efficiency, the model which is utilized in ACC should have only a few parameters and should be simple on the basis that the model can control the vehicle effectively and securely. The IDM is an eligible model which meets these criteria [9].

\subsection{IDM. The IDM is expressed by}

$$
\dot{v}(t)=a_{0}\left[1-\left(\frac{v(t)}{v_{0}}\right)^{4}-\left(\frac{s^{*}(v(t), \Delta v(t))}{s}\right)^{2}\right] .
$$


This expression can be divided into two parts. The first two terms on the right, $\dot{v}_{\text {free }}(t)=a_{0}\left(1-\left(v(t) / v_{0}\right)^{4}\right)$, mean the acceleration when the vehicle is driving on a road without congestion. The last part, $\dot{v}_{\text {brake }}(t)=-a_{0}\left(s^{*} / s\right)^{2}$, means the acceleration which is dominant when the following vehicle approaches the leading vehicle and the following vehicle must decelerate to avoid a rear-end collision

$$
s^{*}(v(t), \Delta v(t))=s_{0}+v(t) T+\frac{v \Delta v}{2 \sqrt{a_{0} b}},
$$

where $s^{*}(v(t), \Delta v(t))$ expresses the desired minimum distance of car-following and is rewritten as $s^{*}$ in the following text. $a_{0}$ is the desired maximum acceleration, $b$ is the desired deceleration, $v(t)$ and $v_{0}$ are the actual speed and desired speed, $\Delta v$ is the speed difference between the following vehicle and the leading vehicle, $s$ is the actual gap, $s_{0}$ indicates that the minimum distance in congested traffic is $2 \mathrm{~m}$, and the desired safe time headway $T$ is equal to $1.5 \mathrm{~s}$ for a car on an uncongested road.

5.2. Dynamic Properties of IDM. IDM was established to simulate the car-following behavior on a freeway [18]. $v_{0}$ takes the value of $120 \mathrm{~km} / \mathrm{h}$ for a car. IDM was analyzed for the four following situations:

(1) equilibrium traffic: in equilibrium traffic of arbitrary density, $\dot{v}(t)=0$ and $\Delta v=0$;

(2) low density: in this situation, $s$ is very large, the term $\left(s^{*} / s\right)^{2}$ of (12) is negligible, and the vehicle speeds up to the desired speed;

(3) braking as a reaction to high approach rates: when a vehicle approaches slower or standing vehicles at a sufficiently high approach rate, the term $s_{0}+v T$ of the desired minimum distance can be neglected;

(4) braking in response to small gaps: the gap $s$ is much smaller than $s^{*}$, but there are no large velocity differences.

In order to directly analyze the IDM, (12) is transformed into

$$
\dot{v}(t)=a_{0}\left[1-\lambda\left(\frac{v(t)}{v_{0}}\right)^{4}-\beta\left(\frac{s^{*}}{s}\right)^{2}\right] .
$$

In the first mode, with $\lambda=0$ and $\beta=1$, the IDM is equivalent to the proportional derivative (PD) control law [19]. In the second mode, with $\lambda=1$ and $\beta=0$, the IDM is similar to the cruise control law, and the motion has nothing to do with the desired time headway $T$ [7]. In the third and fourth modes, the IDM controls deceleration under the range of desired deceleration. In an emergency braking situation, the vehicle also decelerates more strongly than the desired deceleration to avoid a rear-end collision.

In the applicability analysis of IDM, the assumptions are strictly limited $[18,20]$. Nevertheless, the limited assumptions cannot fully represent the actual operation. When the fleet moves at an even and high speed, this situation in which $\dot{v}(t)=0, \Delta v=0$, and $v(t) \rightarrow v_{0}$ is an equilibrium traffic situation. In this situation, (12) should be expressed as $\dot{v}(t)=$ $-a_{0}\left(s^{*} / s\right)^{2}$, and if and only if $s \gg s^{*}$, we have $\dot{v}(t) \rightarrow 0$. However, when the fleet moves with the desired minimum following distance in this situation, $\dot{v}(t)=-a_{0}<0$, which contradicts the precondition $\dot{v}(t)=0$.

In the judgment of car-following behavior, traffic flow theory describes car-following as driving with a gap that is less than $125 \mathrm{~m}$ [21]. Weidemann and Reiter consider that the vehicle continues car-following when the gap is less than $150 \mathrm{~m}$ [22]. We can clearly see that the IDM neglects judgment of car-following behavior. When a vehicle is driving in low density traffic, the vehicle is independent of the vehicle in front and the car-following model becomes invalid. For the ACC system, the computer changes the gap regulation controller into a gap-closing controller when the gap is greater than the gap threshold. Gap control and cruise control are separated to control the vehicle steadily.

5.3. Desired Minimum Distance Modification. The ACC system tends to design a comfortable system, and relatively large headways are applied [23]. CACC achieves smaller headways and more moderate natural driving due to the wireless communication between vehicles [24]. A small headway can improve the road capacity. Therefore, we should try to reduce the headway on the basis of ensuring passenger comfort and safety.

The term $v \Delta v / 2 \sqrt{a_{0} b}$ of the desired minimum distance does not take into account the effect of real-time road surface conditions on the braking distance, and the calculated result is greater than the minimum braking distance. The comfort of passengers is ensured on the basis of avoiding rearend collisions because the greater desired following distance leads to moderate braking. A desired following distance that is smaller than the result of (13) might trigger extreme deceleration during emergency braking. However, the vehicle performs emergency braking only if the leading vehicle performs emergency braking in the actual operation of the vehicles. The probability of emergency braking is relatively small. Consequently, reducing the following gap on the basis of ensuring driving safety to improve the road capacity is a desirable method.

Based on the above analysis, the minimum desired following distance is modified to

$$
s_{1}^{*}=s_{0}+v(t) T+\frac{v^{2}(t)}{2 a_{\max }}-\frac{v_{l}^{2}(t)}{2 a_{l \max }}
$$

where $a_{\max }$ and $a_{l \max }$ present the maximum deceleration of the following vehicle and the leading vehicle. The last two terms of (15) express the minimum distance necessary to avoid a rear-end collision. Taking into account that wireless communication is not mature, we assume that $a_{\max }$ is equal to $a_{l \max }$; that is, $a_{\max }=a_{l \max }$.

In a cut-in scenario, if the minimum desired following distance is calculated by (15), excess adjustment might occur. But the IDM has better performance. These two different results are illustrated in Figure 5. Consequently, in order to guarantee the comfort of passengers, the minimum desired 
following distance model of the IDM is retained by the modified model in the cut-in scenario.

There are two different minimum desired following distance models in the cut-in scenario and the other scenario. Before turning to the minimum desired following distance model, the cut-in scenario should be identified. In this paper, the cut-in scenario is defined as a vehicle changing lane and driving into the middle of a two-vehicle fleet which is moving faster than $6 \mathrm{~m} / \mathrm{s}$. When a vehicle cuts into a two-vehicle fleet, the following gap difference between the subsequent following gap and the original following gap is greater than the possible maximum braking distance in the detection period. Therefore, the control law turning into the cut-in scenario should be given as

$$
s(t-\Delta t)-s(t)>a_{\max } \cdot \Delta t,
$$

where $\Delta t$ presents the detection period. The cut-in scenario stops and the other scenario begins once the actual gap is close to the desired minimum following distance.

5.4. New Car-Following Model. According to the above analysis, (12) is modified as follows:

$$
\dot{v}(t)= \begin{cases}a_{0}\left[1-\left(\frac{v(t)}{v_{0}}\right)^{4}\right], & s>s_{T}, \\ a_{0}\left[1-\left(\frac{s_{1}^{*}}{s}\right)^{2}\right], & s \leq s_{T},\end{cases}
$$

where $s_{T}$ represents the threshold of judging the car-following behavior and is equal to $125 \mathrm{~m}$. When $s$ is smaller than $s_{T}$, the gap controller is triggered, and when the reverse is true, the cruise control is triggered. Equation (17) resolves the contradiction which occurs when a vehicle is moving at an even and high speed. The third and fourth following modes also can be controlled well.

Combining (11), (15), and (17), the new car-following model is expressed as

$$
\begin{aligned}
\dot{v}(t)= & a_{0}\left[1-\left(\frac{s_{1}^{*}}{s}\right)^{2}\right] \\
s_{\prime}^{*}= & s_{0}+v(t) T+k 1\left(\frac{v^{2}(t)}{2 a_{\max }}-\frac{v_{l}^{2}(t)}{2 a_{l \max }}\right) \\
& +k 2 \frac{v \Delta v}{2 \sqrt{a_{0} b}}, \\
a_{\max }= & {\left[\frac{l_{r} u_{f}+l_{f} u_{r}}{L+h\left(u_{f}-u_{r}\right)}\right] * g, }
\end{aligned}
$$

where

$$
\begin{aligned}
& k 1= \begin{cases}1, & \text { others, } \\
0, & \text { cut-in, }\end{cases} \\
& k 2= \begin{cases}0, & \text { others } \\
1, & \text { cut-in. }\end{cases}
\end{aligned}
$$

In the new car-following model, if the actual speed has reached the desired speed, the vehicle will continue to speed up even if $s^{*}$ is smaller than $s$ under the control of (18). This result is not in accordance with reality. To avoid this problem, we add to (18) the constraint that the vehicle maintains a uniform speed even when the result of (18) is a positive number.

5.5. Equilibrium Flow-Density Relation. In equilibrium traffic, $\dot{v}(t)=0, v(t)=v_{l}(t)$, and $a_{\max }=a_{l \max }$. The fleet moves stably with the minimum desired following distance; namely, $s=s_{1}^{*}=s_{0}+v(t) T$. The stable velocity can be presented as $v_{e}=\min \left(v_{o},\left(s-s_{0}\right) / T\right)$. From a macroscopic point of view, the equilibrium traffic can be characterized by the stable traffic flow $Q=\rho v$ as a function of the traffic density $\rho$. According to the relation between gap and density, $1000 / \rho-l=s(l$ represents the length of the vehicle, which is $5 \mathrm{~m}$ in this paper); the relation between flow and density of the new model is shown in Figure 2.

Figure 2(a) shows the relation between flow and density under the premise that the desired speed is $120 \mathrm{~km} / \mathrm{h}$. We can clearly see that the theoretical road capacity of the new model is greater than that of the IDM at the same desired speed and time headway. The theoretical road capacity is equal to the maximum flow. The theoretical capacity of the new model increases with decreasing the time headway. Figure 2(b) shows the relation between gap and density under the premise that the time headway is $1.5 \mathrm{~s}$. We find that the theoretical capacity of the new model increases with increasing the desired speed, but the growth rate is negligible. Therefore, we can conclude that reducing the time headway contributes to improving the road capacity more than improving the desired speed.

\section{Simulation}

In this section, we firstly simulate the new model and the IDM in normal road surface conditions to test the applicability of the new model. Simulations of the new model and the IDM in changing road surface conditions are also carried out to compare the performance of the new model and IDM in real-time road surface conditions. All of the simulations are conducted with MATLAB/Simulink.

6.1. Car-Following Properties in Normal Road Surface Conditions. We carried out two simulations in normal road surface conditions. The first experiment aims to test and contrast the stabilities of the new model and IDM in normal traffic. The second experiment aims to test and contrast the stabilities of the new model and IDM in the cut-in scenario. In these two simulations, the vehicles are driving on a dry asphalt road and the difference between tires is ignored. We assume that the maximum deceleration is $7 \mathrm{~m} / \mathrm{s}^{2}$.

(1) Normal Traffic. In normal traffic, to observe the carfollowing behavior, a four-vehicle fleet is used and moves at high speed. In the first cycle, the leading vehicle drives at $25 \mathrm{~m} / \mathrm{s}$ for $20 \mathrm{~s}$ and then accelerates at a constant acceleration 


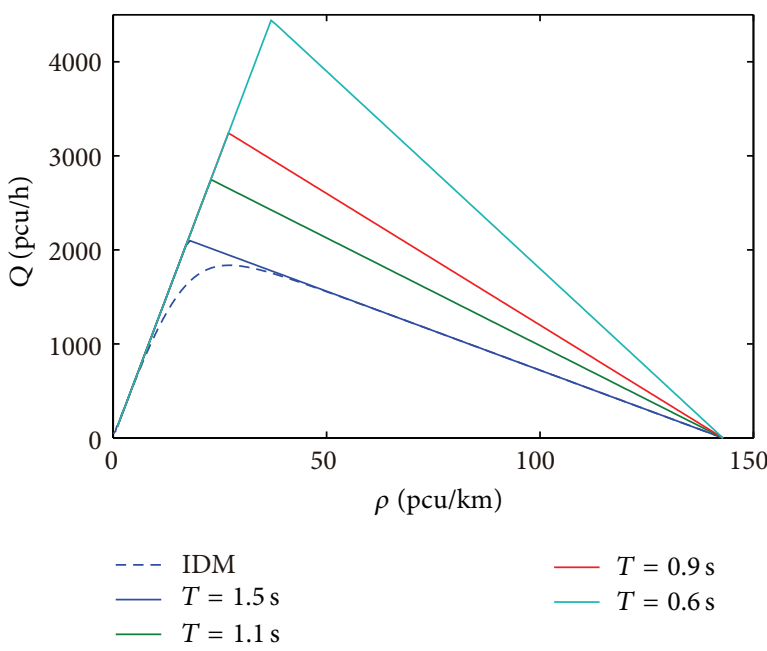

(a)

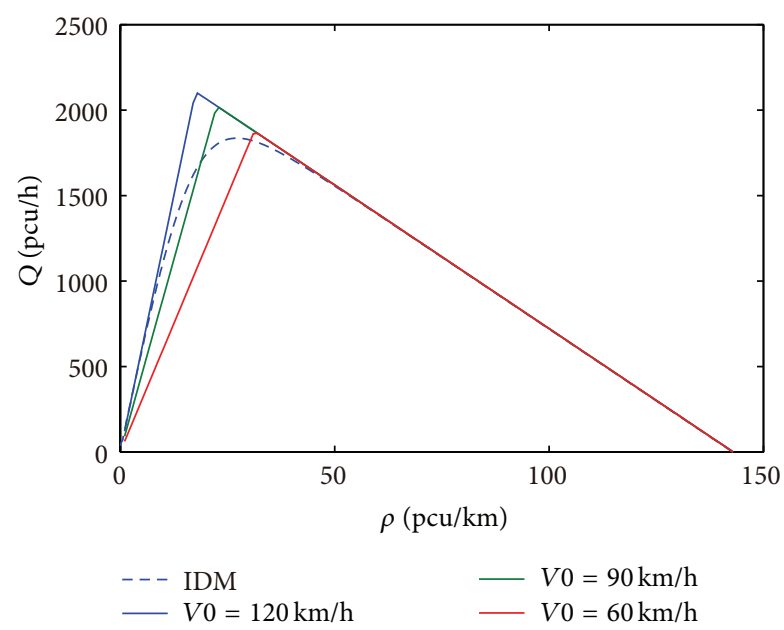

(b)

FIgURE 2: Equilibrium flow-density relation.

of $0.25 \mathrm{~m} / \mathrm{s}^{2}$ to reach $30 \mathrm{~m} / \mathrm{s}$. The vehicle decelerates at a constant deceleration of $0.25 \mathrm{~m} / \mathrm{s}^{2}$ to return to $25 \mathrm{~m} / \mathrm{s}$ and remains at a constant speed for $20 \mathrm{~s}$. In the second cycle, it accelerates at a constant acceleration of $0.33 \mathrm{~m} / \mathrm{s}^{2}$ to reach $30 \mathrm{~m} / \mathrm{s}$ and maintains a constant speed for $20 \mathrm{~s}$. Then, the vehicle decelerates at a constant deceleration of $0.5 \mathrm{~m} / \mathrm{s}^{2}$ to return to $25 \mathrm{~m} / \mathrm{s}$ and maintains a constant speed for $15 \mathrm{~s}$. In the third cycle, it accelerates at a constant acceleration of $0.5 \mathrm{~m} / \mathrm{s}^{2}$ to reach $30 \mathrm{~m} / \mathrm{s}$ and maintains a constant speed for $10 \mathrm{~s}$. Then, the vehicle decelerates at a constant deceleration of $1 \mathrm{~m} / \mathrm{s}^{2}$ to return to $25 \mathrm{~m} / \mathrm{s}$ and maintains a constant speed for $10 \mathrm{~s}$. In the last cycle, it accelerates at a constant acceleration of $1 \mathrm{~m} / \mathrm{s}^{2}$ to reach $30 \mathrm{~m} / \mathrm{s}$ and maintains a constant speed for $20 \mathrm{~s}$. The three other following vehicles run with the new model control and IDM control. For the comparison of the new model and IDM, the same desired speed $(120 \mathrm{~km} / \mathrm{h})$ and the same desired safe time headway $(1.5 \mathrm{~s})$ are used. At the beginning of the fleet movement, the four vehicles have the same initial speed of $25 \mathrm{~m} / \mathrm{s}$. And the three following vehicles have the same following gap of $39.5 \mathrm{~m}$.

Figures 3 and 4 show the car-following with the IDM control and new model control. It can be found from Figures 3 (a) and 4(a) that the response time of the IDM is longer than that of the new model. The longer response time means the following vehicle must maintain a sufficient gap to avoid a rear-end collision. This result is consistent with Figures 3(c) and 4(c). The IDM keeps the actual time headway of the fleet around $2.1 \mathrm{~s}$ compared with $1.6 \mathrm{~s}$ in the new model. The longer response time of the IDM controller determines that the vehicle needs a larger car-following gap and time headway, but its corresponding acceleration and deceleration are milder than those of the new model controller. It can be found from Figures 3(b) and 4(b). Table 1 shows the parameters selected from the new model and the IDM controller. We can find that the scope of acceleration and deceleration of the new model controller is greater than that of the IDM controller, but it is in the acceptable range and the comfort of passengers is still good. Consequently, the new
TABLE 1: Selected parameters of the new model and the IDM controller.

\begin{tabular}{ccccc}
\hline & & Headway $(\mathrm{s})$ & Gap $(\mathrm{m})$ & $\begin{array}{c}\text { Deceleration } \\
\left(\mathrm{m} / \mathrm{s}^{2}\right)\end{array}$ \\
\hline \multirow{2}{*}{ AVG } & $\begin{array}{c}\text { New } \\
\text { model }\end{array}$ & 1.60 & 43.67 & -0.21 \\
& IDM & 2.11 & 57.12 & -0.17 \\
\hline \multirow{2}{*}{ MAX } & $\begin{array}{c}\text { New } \\
\text { model } \\
\end{array}$ & 1.84 & 56.60 & -1.05 \\
& IDM & 2.49 & 74.05 & -0.70 \\
\hline
\end{tabular}

model is suitable and more effective than the IDM from the capacity point of view.

(2) Cut-In Scenario. When two vehicles are driving in the same lane and other vehicles want to enter the lane between them, sudden and unexpected cut-ins will occur in a short period of time. The sudden decrease of the following gap should lead to extreme deceleration for safety. Consequently, the braking efficiency should be considered to test the performance of the new model and the IDM in the cut-in scenario. For the sake of clarity, a two-vehicle fleet response in the cut-in scenario is shown in Figure 5.

The leading vehicle and the following vehicle have the same initial speed of $25 \mathrm{~m} / \mathrm{s}$ and the initial gap is $40 \mathrm{~m}$. The leading vehicle is driving at a constant speed $(25 \mathrm{~m} / \mathrm{s})$ and the following vehicle is tracking the leader's speed. Around the 60th second, a cut-in vehicle merges between them, and the following vehicle and the new leading vehicle make up a new two-vehicle fleet. To demonstrate the necessity of cutin judgment, the minimum desired following distance model of the other scenario, which is also shown in Figure $5(k 1=$ $1, k 2=0)$, is simulated in the cut-in scenario.

It is obvious that the IDM has the best performance. The peak of the following deceleration of the IDM is near $1.9 \mathrm{~m} / \mathrm{s}^{2}$. In the new model $(k 1=0, k 2=1)$, when the following 


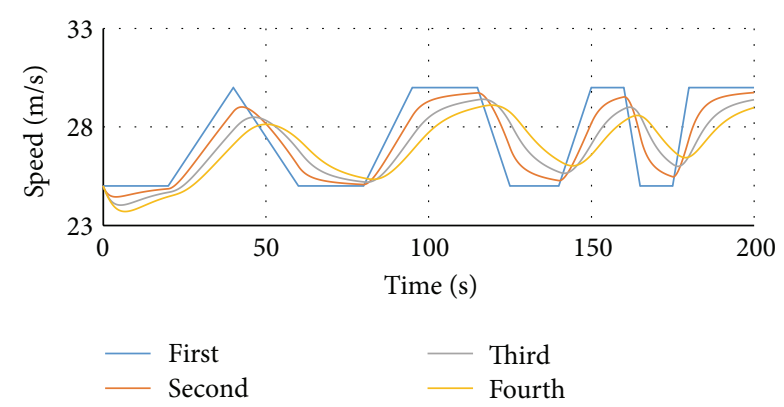

(a)

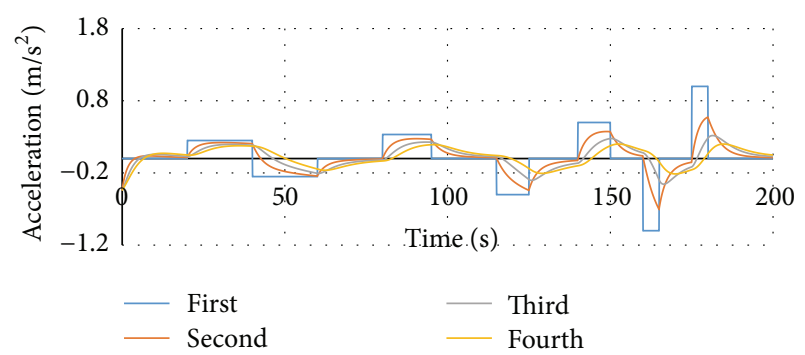

(b)

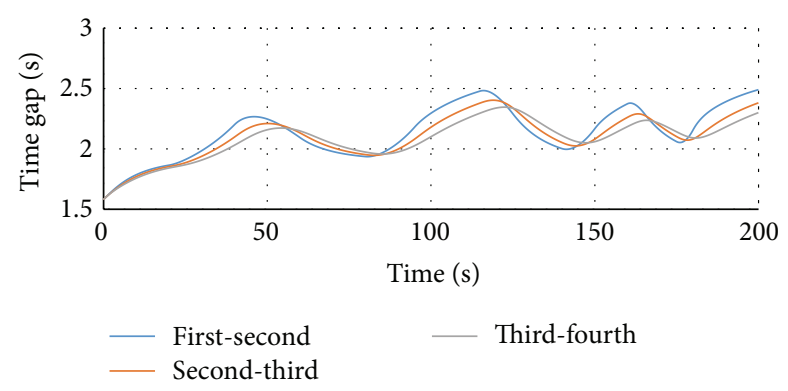

(c)

FIGURE 3: Four-vehicle car-following properties with IDM control in normal traffic.

vehicle detects the cut-in vehicle, the following vehicle brakes to increase the gap between itself and the new leading vehicle. The peak deceleration of the following vehicle in the new model is near $4.3 \mathrm{~m} / \mathrm{s}^{2}$ and is greater than that in the IDM, but it is in the range of acceptable deceleration. The new model has smaller following gap than IDM; therefore, the vehicle controlled by new model brakes stronger to avoid a rear-end collision. However, the probability of cut-in scenario is small, because the small following gap of the new model does not provide chance for other vehicles to cut in.

By comparing the gray solid line $(k 1=0, k 2=1)$ with the yellow solid line $(k 1=1, k 2=0)$, we find that the peak decelerations are equal. However, the model whose minimum desired following distance model in the cutin scenario is replaced by the others has the disadvantage of excess adjustment. So, we can conclude that applying a different minimum desired following distance model for new model is necessary and suitable.

6.2. Car-Following Properties in Changing Road Surface Conditions. The real-time maximum deceleration varies with the

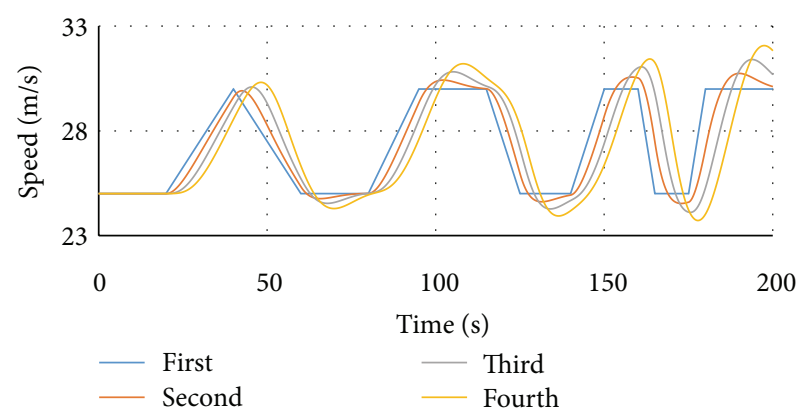

(a)

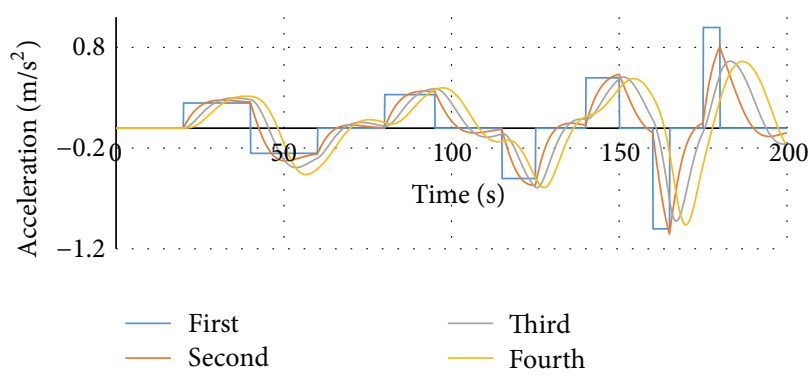

(b)

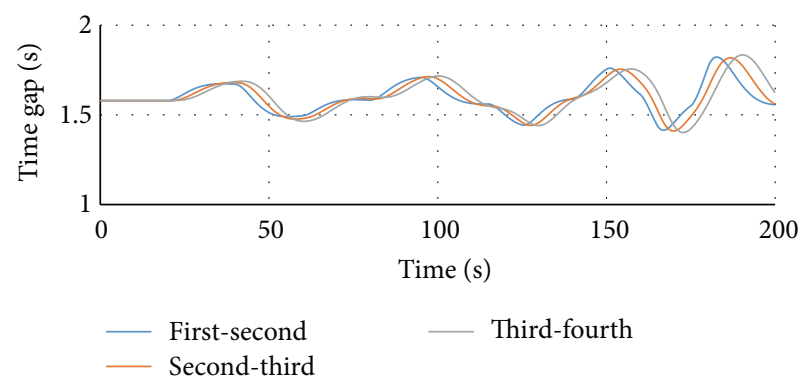

(c)

FIGURE 4: Four-vehicle car-following properties with new model control in normal traffic.

real-time road surface condition. Consequently, the parameter $a_{\max }$ is different on diverse road surfaces. The emergency braking distance is dependent on $a_{\max }$. On the good road surface (dry asphalt road), the emergency braking distance is relatively short because of the large $a_{\max }$. However, the emergency braking distance on the bad road surface (icy road) is longer than the good road surface because of the smaller $a_{\max }$. Therefore, the driver can maintain a greater following gap on the bad road surface.

The IDM considers the real-time road conditions by adjusting the desired time headway. The minimum desired following distance in the new model is a function of the real-time maximum deceleration. To test the performance of the IDM and the new model in real-time road surface conditions, a simulation is carried out. In the simulation, two vehicles move from a dry asphalt road to an icy road at $190 \mathrm{~m}$. The total length of the road section is $530 \mathrm{~m}$. The maximum decelerations on the dry asphalt road and the icy road are 7 and $1.764 \mathrm{~m} / \mathrm{s}^{2}$. The leading vehicle is driven by a human, and the driver slows down before moving onto the icy road because the human driver can see the condition of the road in 


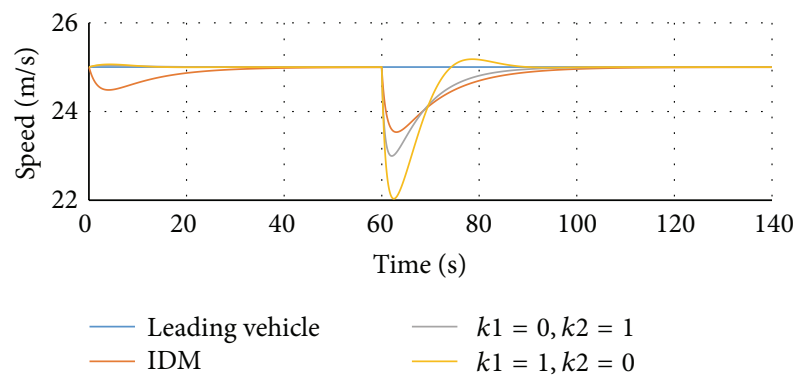

(a)

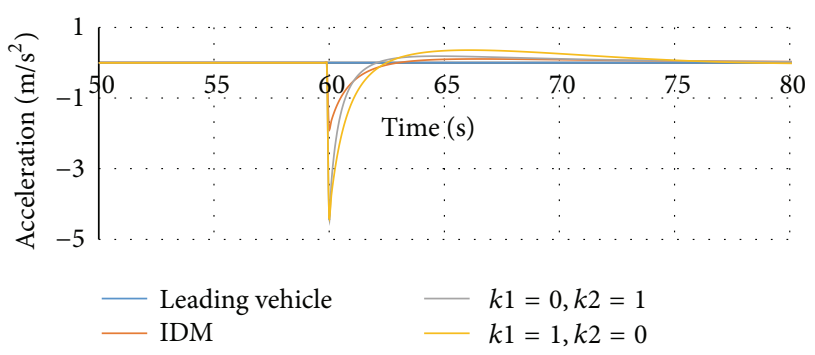

(b)

Figure 5: Cut-in response.

front and the front vehicle of the leading vehicle brakes before the leading vehicle drives onto the icy road.

The leading vehicle moves onto the icy road at $13.6 \mathrm{~s}$. In the IDM control, the driver manually changes the desired time headway from 1.5 to $2.2 \mathrm{~s}$ after observing the change in the road condition. The sudden change in the minimum desired following distance of the IDM which is shown in Figure 6(c) $\left(\right.$ IDM- $\left.{ }^{*}\right)$ is attributed to the change in the desired time headway. However, the desired time headway is a constant value in the new model. The driver does not need to manually change the desired time headway. The change in the real-time maximum deceleration represents the change in the real-time road condition.

In the new model control, the maximum deceleration is updated after the change in the road condition is detected by the sensors. Because the minimum desired following distance of the new model is a function of the real-time maximum deceleration, it also changes suddenly with changes in the real-time maximum deceleration. From Figure 6(c), we can find the sudden change (new model- $s^{*}$ ). The sudden change of the new model is smaller than that of the IDM. Consequently, the new model controller is more stable and decelerates more smoothly than the IDM controller. The result is in accordance with Figure 6(b). The maximum deceleration of the IDM is $-2.95 \mathrm{~m} / \mathrm{s}^{2}$ and the maximum deceleration of the new model is $-2.51 \mathrm{~m} / \mathrm{s}^{2}$.

Comparing the response time of the following vehicle of the new model with that of the IDM after the vehicles drive onto an icy road, we find that the new model controller has greater delay than the IDM controller according to Figure 6(a). The reason is that the desired time headway is adjusted manually by the human driver before the vehicle drives onto the icy road. However, the parameters of the new model are adjusted automatically when the vehicle drives onto the icy road.

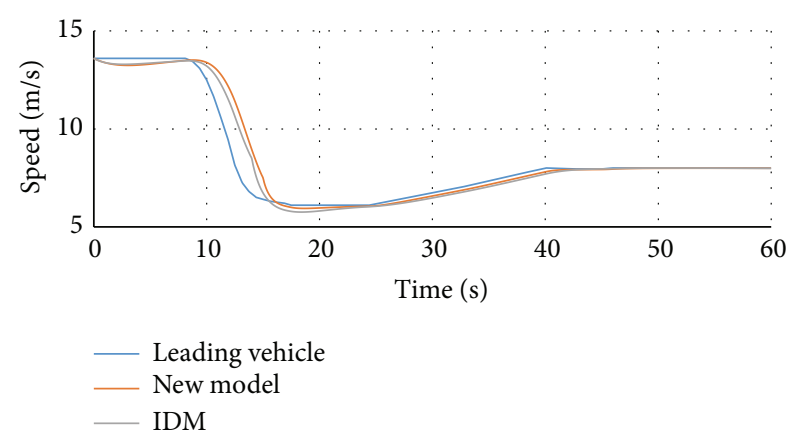

(a)

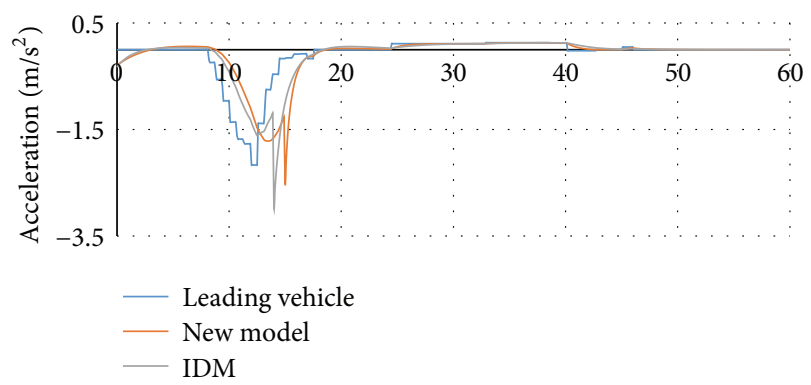

(b)

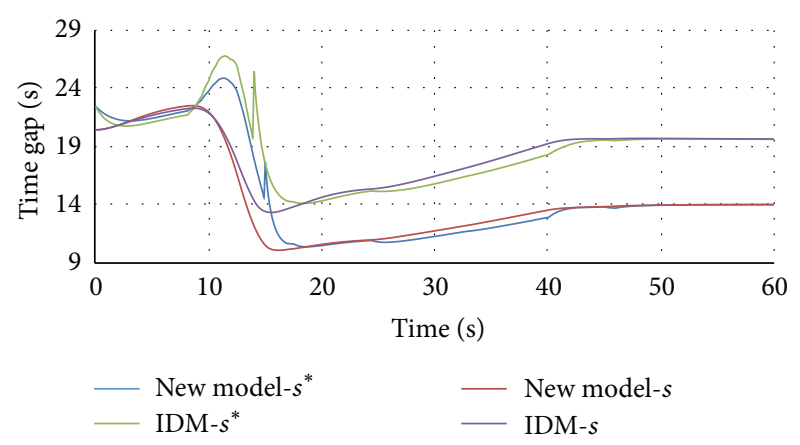

(c)

FIgURE 6: The car-following properties on a changing road.

\section{Conclusion}

In this paper, the real-time maximum deceleration estimation approach is introduced. We analyzed the IDM and found a disadvantage which could occur when the vehicle moves at high and even speed. According to the disadvantage and the real-time maximum deceleration, a new car-following model is established on the basis of the IDM. The minimum desired following distance of the new model contains minimum collision-avoidance distance and ensures the safety of the following vehicles.

The equilibrium flow-density relations of the new model and the IDM are compared and the result indicates that the new model controller improves road capacity more than the IDM controller and that reducing the time headway is more efficient for improving the road capacity than increasing the desired speed.

The new model can keep the vehicle moving with smaller time headway than the IDM on the basis of ensuring safety 
and comfort according to the simulation of equilibrium traffic on a normal road. The smaller time headway means greater road capacity which can alleviate the traffic jam. Moreover, the peak of actual deceleration of the new model may be roughly greater than that of IDM in the cut-in scenario, but it is within the acceptable range. In changing road conditions, the new model can have smaller deceleration than the IDM and the controlled vehicle decelerates smoothly. Consequently, considering the effect of real-time maximum deceleration on car-following not only can improve the driving comfort and safety but also can alleviate the traffic jam which has important significance for sustainable transportation.

\section{Conflict of Interests}

The authors declare that they have no competing financial interests.

\section{Acknowledgment}

The authors would like to thank the reviewers for their careful reading and for providing some pertinent suggestions.

\section{References}

[1] B. Li, H. Du, and W. Li, "Comparative study of vehicle tyreroad friction coefficient estimation with a novel cost-effective method," Vehicle System Dynamics, vol. 52, no. 8, pp. 1066-1098, 2014.

[2] Z. P. Yu, J. L. Zuo, and L. J. Zhang, "A summary on the development status quo of tire-road friction coefficient estimation techniques," Automotive Engineering, vol. 28, no. 6, pp. 546-549, 2006.

[3] X. B. Fan and P. Deng, "Study on the tire/road friction coefficient estimation," Auto Engineer, vol. 12, pp. 47-50, 2013.

[4] R. Rajamani, N. Piyabongkarn, J. Lew et al., "Tire-road frictioncoefficient estimation," IEEE Control Systems Magazine, vol. 30, no. 4, pp. 54-69, 2010.

[5] R. Rajamani, G. Phanomchoeng, D. Piyabongkarn, and J. Y. Lew, "Algorithms for real-time estimation of individual wheel tire-road friction coefficients," IEEE/ASME Transactions on Mechatronics, vol. 17, no. 6, pp. 1183-1195, 2012.

[6] D.-H. Wang and S. Jin, "Review and outlook of modeling of car following behavior," China Journal of Highway and Transport, vol. 25, no. 1, pp. 115-127, 2012.

[7] V. Milanes, S. E. Shladover, J. Spring, C. Nowakowski, H. Kawazoe, and M. Nakamura, "Cooperative adaptive cruise control in real traffic situations," IEEE Transactions on Intelligent Transportation Systems, vol. 15, no. 1, pp. 296-305, 2014.

[8] T. Dijker, P. H. L. Bovy, and R. G. M. M. Vermijs, "Car-following under congested conditions: empirical findings," Transportation Research Record, no. 1644, pp. 20-28, 1998.

[9] A. Kesting, M. Treiber, M. Schönhof, and D. Helbing, "Adaptive cruise control design for active congestion avoidance," Transportation Research Part C: Emerging Technologies, vol. 16, no. 6, pp. 668-683, 2008.

[10] I. Soria, L. Elefteriadou, and A. Kondyli, "Assessment of carfollowing models by driver type and under different traffic, weather conditions using data from an instrumented vehicle,"
Simulation Modelling Practice and Theory, vol. 40, pp. 208-220, 2014.

[11] T. Q. Tang, Y. P. Wang, X. B. Yang, and Y. H. Wu, "A new car-following model accounting for varying road condition," Nonlinear Dynamics, vol. 70, no. 2, pp. 1397-1405, 2012.

[12] T. Q. Tang, J. G. Li, H. J. Huang, and X. B. Yang, "A carfollowing model with real-time road conditions and numerical tests," Measurement, vol. 48, no. 1, pp. 63-76, 2014.

[13] K. Yi and J. Taeyoung, "Observer based estimation of tireroad friction for collision warning algorithm adaptation," JSME International Journal, Series C: Dynamics, Control, Robotics, Design and Manufacturing, vol. 41, no. 1, pp. 116-124, 1998.

[14] D. Y. Qu, X. F. Chen, W. S. Yang, and X. H. Bian, "Modeling of car-following required safe distance based on molecular dynamics," Mathematical Problems in Engineering, vol. 2014, Article ID 604023, 7 pages, 2014.

[15] L. H. Xu, Q. Luo, J. W. Wu, and Y. G. Huang, "Study of carfollowing model based on minimum safety distance," Journal of Highway and Transportation Research and Development, vol. 10, no. 10, pp. 95-100, 2010.

[16] R. Hoseinnezhad and A. Bab-Hadiashar, "Efficient antilock braking by direct maximization of tire-road frictions," IEEE Transactions on Industrial Electronics, vol. 58, no. 8, pp. 35933600, 2011.

[17] Z. H. Wang and S. M. Shao, "Simulation of adaptive cruise control algorithm of vehicle," Computer Engineering and Design, vol. 2, pp. 604-608, 2014.

[18] M. Treiber, A. Hennecke, and D. Helbing, "Congested traffic states in empirical observations and microscopic simulations," Physical Review E: Statistical Physics, Plasmas, Fluids, and Related Interdisciplinary Topics, vol. 62, no. 2, pp. 1805-1824, 2000.

[19] P. Zheng and M. McDonald, "Manual vs. adaptive cruise control-can driver's expectation be matched?" Transportation Research Part C: Emerging Technologies, vol. 13, no. 5-6, pp. 421431, 2005.

[20] D. Helbing, A. Hennecke, V. Shvetsov, and M. Treiber, "Microand macro-simulation of freeway traffic," Mathematical and Computer Modelling, vol. 35, no. 5-6, pp. 517-547, 2002.

[21] N. H. Gartner, C. J. Messer, and A. K. Rathi, "Traffic flow theory (update of TRB special report 1165)," Tech. Rep., TRB, Washington, DC, USA, 1997.

[22] R. Weidemann and U. Reiter, "Microscopic traffic simulation. The simulation system-mission," Project ICARUS (V1052) Final Report, University Karlsruhe, 1992.

[23] J. Ploeg, B. T. M. Scheepers, E. van Nunen, N. van de Wouw, and H. Nijmeijer, "Design and experimental evaluation of cooperative adaptive cruise control," in Proceedings of the 14th International IEEE Conference on Intelligent Transportation Systems (ITSC '11), pp. 260-265, IEEE, Washington, DC, USA, October 2011.

[24] F. Bu, H. Tan, and J. Huang, "Design and field testing of a cooperative adaptive cruise control system," in Proceedings of the American Control Conference (ACC '10), pp. 4616-4621, IEEE, Baltimore, Md, USA, June 2010. 


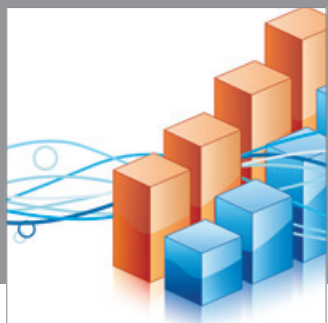

Advances in

Operations Research

mansans

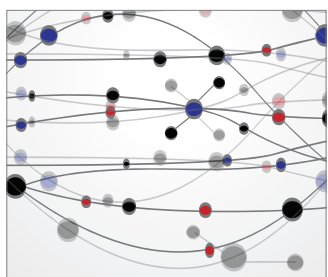

The Scientific World Journal
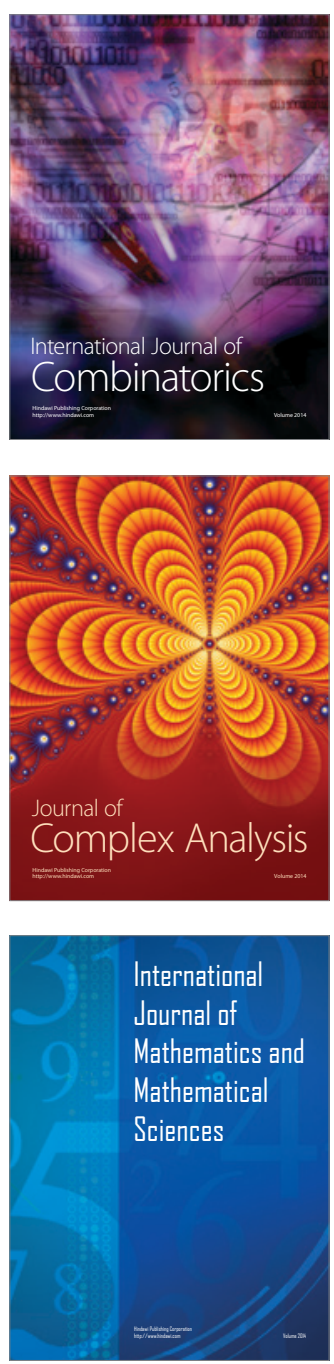
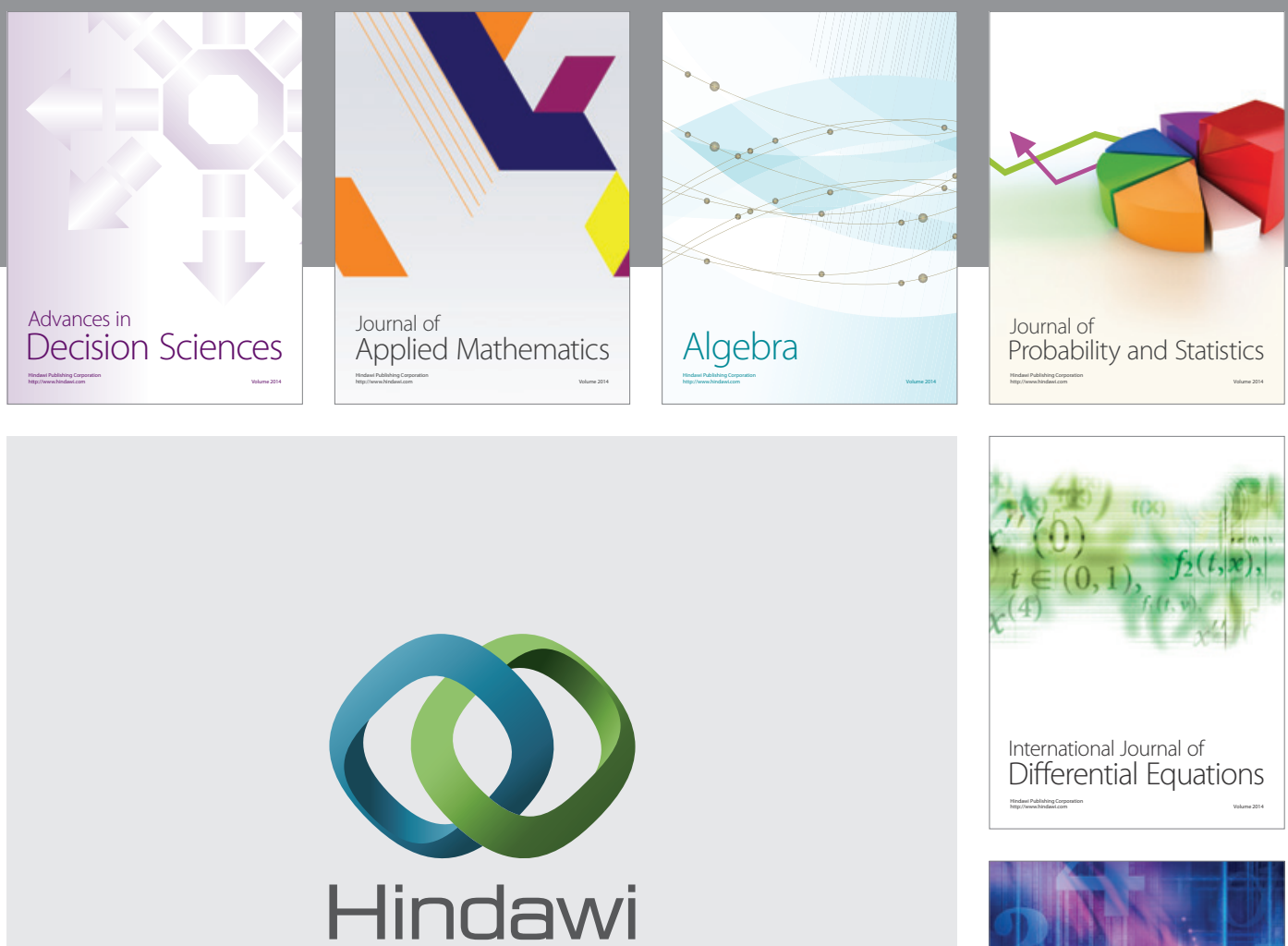

Submit your manuscripts at http://www.hindawi.com
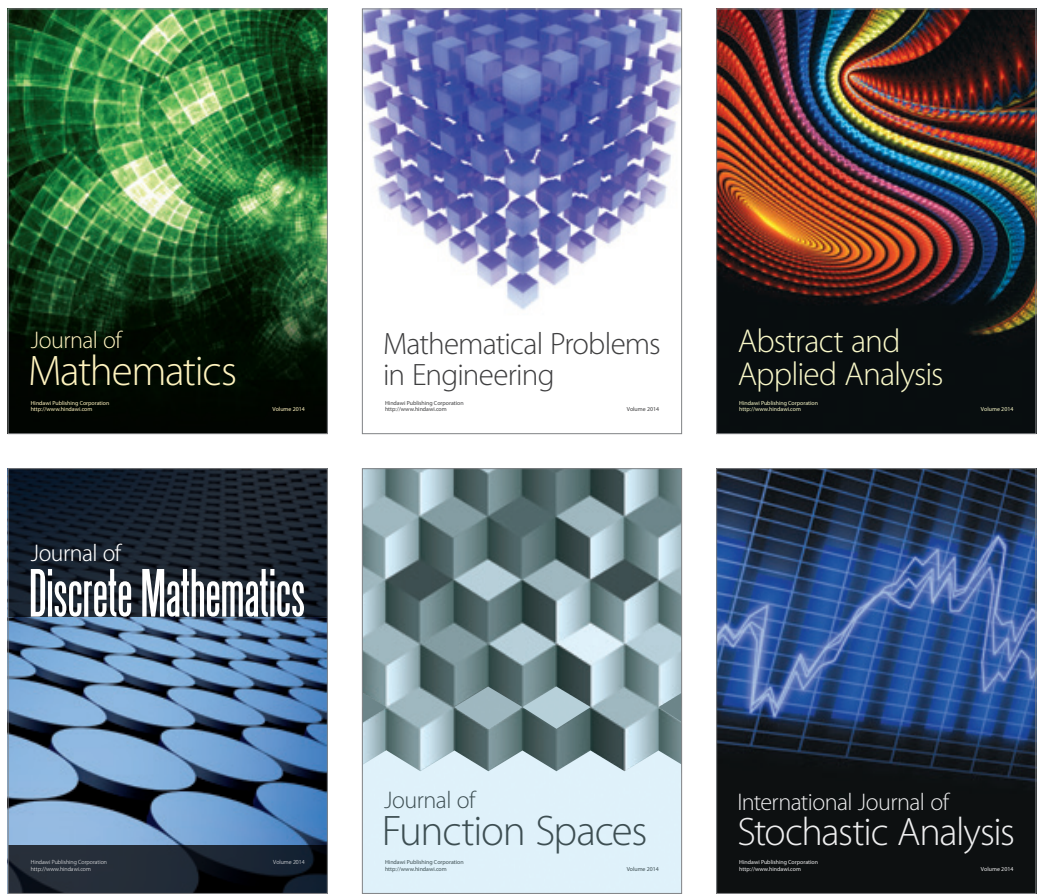

Journal of

Function Spaces

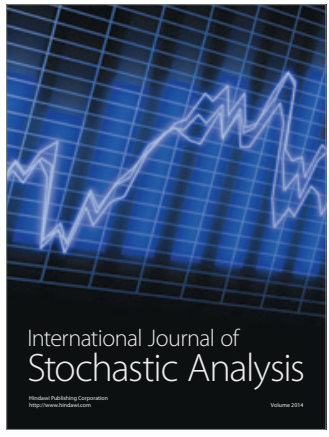

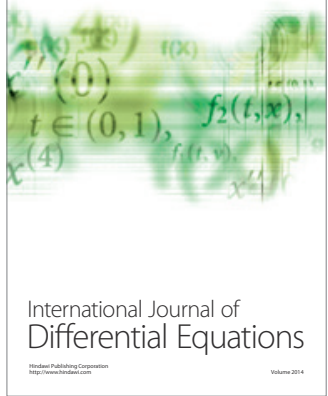
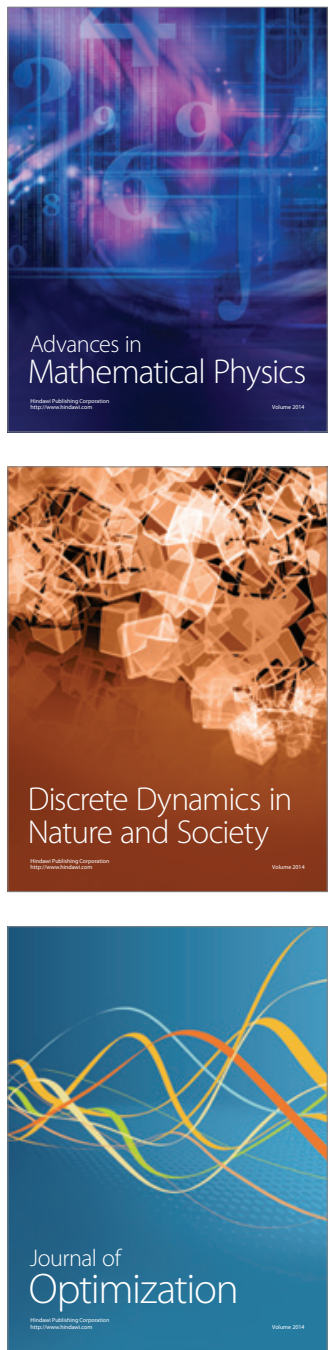